\title{
Magnetometric resistivity tomography using chaos polynomial expansion
}

\author{
M. T. Vu ${ }^{\oplus,}{ }^{1}$ A. Jardani, ${ }^{1}$ A. Revil ${ }^{2}$ and M. Jessop ${ }^{3}$ \\ ${ }^{1}$ Université de Rouen, M2C, UMR 6143, CNRS, Morphodynamique Continentale et Côtière, 76821 Mont Saint Aignan, France. E-mail: \\ minh-tan.vu@univ-rouen.fr \\ ${ }^{2}$ Université Grenoble Alpes, USMB, CNRS, EDYTEM, 73000 Chambéry, France \\ ${ }^{3}$ Willowstick Technologies, LLC; 132 East 13065 South, Draper, UT 84020, USA
}

Accepted 2020 February 7. Received 2020 February 4; in original form 2019 October 11

\begin{abstract}
S UMMAR Y
We present an inversion algorithm to reconstruct the spatial distribution of the electrical conductivity from the analysis of magnetometric resistivity (MMR) data acquired at the ground surface. We first review the theoretical background of MMR connecting the generation of a magnetic field in response to the injection of a low-frequency current source and sink in the ground given a known distribution of electrical conductivity in the subsurface of the Earth. The forward modelling is based on sequentially solving the Poisson equation for the electrical potential distribution and the magnetostatic (Biot and Savart) equation for the magnetic field. Then, we introduce a Gauss-Newton inversion algorithm in which the logarithm of the electrical conductivity field is parametrized by using the chaos polynomial expansion in order to reduce the number of model parameters. To illustrate how the method works, the algorithm is successfully applied on four synthetic models with 3-D heterogeneous distribution of the electrical conductivity. Finally, we apply our algorithm to a field case study in which seepage was known to be occurring along an embankment of a headrace channel to a power station.
\end{abstract}

Key words: Hydrogeophysics; Electromagnetic theory; Magnetic anomalies: modelling and interpretation; Inverse theory; Numerical modelling.

\section{INTRODUCTION}

The characterization of the shallow subsurface in terms of electrical conductivity/resistivity distribution can be done with galvanometric and induction-based methods (e.g. Binley \& Kemna 2005; Friedman 2005; Samouëlian et al. 2005; Florsch et al. 2012). Electrical conductivity can be used to characterize the texture of porous soils and rocks (Waxman \& Smits 1968; Vinegar \& Waxman 1984). Electrical resistivity tomography (ERT) is one of the most popular methods in the field of hydrogeophysics. These investigations concern for instance the detection of the presence and development of sinkholes (e.g. Jardani et al. 2006; Ezersky et al. 2013; Redhaounia et al. 2016), the delineation of a contaminated aquifer (e.g. Abbas et al. 2018; Grünhut et al 2018; Liao et al. 2018) and the mapping of sea intrusion in coastal areas (e.g. George et al. 2004; De Franco et al. 2009; Kazakis et al. 2016).

Galvanometric ERT is easy to perform partly because the sensors (stainless steel electrodes) are simple and cheap. In addition, both field multichannel resistivity meter and data processing software are available (Loke \& Barker 1996; Günther 2007; Karaoulis et al. 2011). Resistivity can also be obtained using a less-known technique called the magnetometric resistivity (MMR) method. Earlier works
(Jakosky 1933) have established the theoretical foundations of this method, which is based on the injection/retrieval of a low-frequency electrical current in the ground between a pair of electrodes A and $\mathrm{B}$ and measuring the three components of the magnetic field $B_{x}, B_{y}$ and $B_{z}$ by using a magnetometer. The measured magnetic field is the result of three contributions (Nabighian 1984). The first is due to the wires used for the current injections and connected to the power generator generally at the ground surface. The second contribution called the primary field is assigned to the injected current at the ground surface, which depends only on the intensity of the injected current and the distance separating the current electrodes A and $\mathrm{B}$ and the observation stations located in between. The third contribution is associated with the contrast (gradient) in the electrical conductivity of the ground. In order to isolate this last contribution associated with subsurface heterogeneities, the first two contributions need to be computed from the Biot and Savart equation and removed from the total measured magnetic field distribution (Edwards 1974; Edwards \& Howell 1976). Then, the residual magnetic field is used in an inverse algorithm to reconstruct the heterogeneity of the electrical conductivity field. In that respect, Chen et al. (2002) implemented a Gauss-Newton algorithm to image the 3-D electrical conductivity of the subsurface. LaBrecque et al. (2003) developed 
a joint interpretation of the electrical (potential) and magnetic resistivity data. Kulessa et al. (2002) used magnetometric resistivity to locate the plume of a solute in a saturated sand during a sandbox experiment. Finally, Jessop et al. (2018) presented a new approach to interpret MMR data, which relies on the reconstruction of the electrical current density crossing the conductive media between two current electrodes A and B instead of the electrical conductivity distribution itself. This approach has been used to detect the conductive anomalies associated with groundwater pathways in mine rock dumps.

In this paper, we propose a new inverse formulation to handle the MMR data in order to determine the 3-D electrical conductivity contribution. The approach relies on a Gauss-Newton algorithm in which the electrical conductivity is parametrized with the chaos polynomial expansion technique. In the past few decades, the polynomial expansion methods were found to be an attractive and promising tool to approximate the forward solution of stochastic problems in engineering (Ghanem 1998; Ghanem et al. 2000; Le Mâtre et al. 2001, 2002; Xiu et al. 2001; Debusschere et al. 2003; Reagan et al. 2004) and were extended to solve the inverse problems (Marzouk et al. 2007; Abbasi \& Gholami 2017). This type of parametrization permits to significantly reduce the number of model parameters required to estimate and enables solution convergence in less time. Some similar ideas have been discussed in the literature in which the projection on the basis of interpolated functions is adopted to interpolate meshing nodes, such as linear interpolations and B-splines (de Boor 1978; Fomel 2000). Applications of these approaches have been performed in the field of seismicity (Farra \& Madariaga 1988; Pereyra 1996; Zhang \& Wang 2010). These methods based on representing the interpolated function by piecewise polynomials can be considered as local approaches. In this study, we attempt to work with a distinct global representation of the model parameter representation, rarely applied in geophysical studies to predict physical properties with less computation demand in the inverse process (Abbasi \& Gholami 2017). The approach is validated with synthetic cases and is applied to a real case study.

\section{THEORETICAL BACKGROUND}

\subsection{MMR}

MMR is an active geophysical method in which a magnetic field is measured in response to the injection of a low-frequency current between two electrodes A and B set up at the ground surface. This magnetic field is associated with the electric current originating from the connecting wires (connected to the current generator at the ground surface), the injection current on a conductive homogeneous subsurface and the relative contrasts in the distribution (gradients) of the electrical conductivity field. The numerical simulation of this phenomenon involves the Maxwell system of equations in their quasi-static approximation (Chen et al. 2002; Chen \& Oldenburg 2006):

$\nabla \times \boldsymbol{E}=0$,

$\nabla \cdot \boldsymbol{B}=0$

$\nabla \times \boldsymbol{B}=\mu \boldsymbol{J}_{c}$,

where $\boldsymbol{E}$ is the electric field (in $\mathrm{V} \mathrm{m}^{-1}$ ), $\boldsymbol{B}=\mu \boldsymbol{H}$ denotes the magnetic flux density (in T), $\boldsymbol{H}$ is the magnetic field (in $\mathrm{A} \mathrm{m}^{-1}$ ), $\mu$ is the magnetic permeability of free space $\left(4 \pi \times 10^{-7} \mathrm{~T} \mathrm{~m} \mathrm{~A}^{-1}\right)$ and $\boldsymbol{J}_{c}$ denotes the conduction current density (in $\mathrm{A} \mathrm{m}^{-2}$ ).

For a low-frequency electrical field, eq. (1) implies that the electrical field can be derived from a scalar potential $\varphi$ (in V) by

$\boldsymbol{E}=-\nabla \varphi$.

Induction is therefore neglected. The total current in the medium can be decomposed into two components: a primary current flow related to the source current density $\boldsymbol{J}_{s}$ (in $\mathrm{Am}^{-1}$ ) and a volume current flow due to the electrical field in the medium:

$\boldsymbol{J}_{c}=\sigma \boldsymbol{E}+\boldsymbol{J}_{s}=-\sigma \nabla \varphi+\boldsymbol{J}_{s}$,

where $\sigma$ denotes the electric conductivity (in $\mathrm{S} \mathrm{m}^{-1}$ ).

The conservation law for the charge is given by

$\nabla \cdot \boldsymbol{J}_{c}=0$,

$\nabla \cdot(\sigma \nabla \varphi)=\nabla \cdot \boldsymbol{J}_{s}$.

Using eq. (5) with eq. (7) yields the well-known equation where the source of current density $\boldsymbol{J}_{s}$ can be obtained by solving

$\nabla \cdot \boldsymbol{J}_{s}=I\left(\delta\left(\boldsymbol{r}-\boldsymbol{r}_{\mathrm{A}}^{+}\right)-\delta\left(\boldsymbol{r}-\boldsymbol{r}_{\mathrm{B}}^{+}\right)\right)$,

where $\boldsymbol{r}$ denotes the position vector, $\boldsymbol{r}_{\mathrm{A}}^{+}$and $\boldsymbol{r}_{\mathrm{B}}^{+}$represent the positions of the current source and sink respectively for the current electrodes A and B, $I$ (in A) denotes the current and $\delta$ is the Dirac distribution.

Eqs (7) and (8) can be reformulated as

$\nabla \cdot(\sigma \nabla \varphi)=I\left(\delta\left(\boldsymbol{r}-\boldsymbol{r}_{\mathrm{A}}^{+}\right)-\delta\left(\boldsymbol{r}-\boldsymbol{r}_{\mathrm{B}}^{+}\right)\right)$.

This partial differential equation can be solved numerically with an appropriate boundary condition to obtain the potential distribution $\varphi$. Once the electrical potential field is solved, the magnetic field can be determined by solving

$\nabla \times \boldsymbol{B}=\mu \boldsymbol{J}_{c}=\mu\left(-\sigma \nabla \varphi+\boldsymbol{J}_{s}\right)$.

We solve below such a forward problem using a partial differential equation solver, namely COMSOL, based on the finite-element method. The work is done in 3-D for the case studies discussed hereafter.

\subsection{Parametrization: polynomial expansion}

The inverse problem in MMR is often challenging since the problem is nonlinear between the magnetic field and the electrical conductivity field. It is also ill-conditioned and underdetermined (Chen et al. 2002). Particularly, when the inverse problem is formulated in the large-scale scheme with a large number of model parameters to estimate, it requires imposing a suitable regularization constraint for targeting a plausible and realistic solution (Kitanidis 1998). In this approach, we aim to develop an overdetermined formulation using a polynomial expansion that will significantly reduce the number of model parameters. The most important advantage of the polynomial expansion approach is that the transformation can provide an estimation of the field within a desired precision without using a huge number of terms (Marzouk et al. 2007).

The logarithm of electrical conductivity field can be parametrized with the polynomial expansion (Wiener 1938; Xiu \& Karniadakis 2002):

$s=\sum_{i, j, k=0}^{\infty} a_{i j k} \Psi_{i}(x) \Psi_{j}(y) \Psi_{k}(z)$, 
where $s=-\log _{10} \sigma, a_{i j k}$ are the coefficients and $\Psi_{i}(x)$ is the orthogonal polynomial of degree $i$ corresponding to axis $x$, and similarly for $\Psi_{j}(y)$ and $\Psi_{k}(z)$. We use the logarithm of the conductivity field as a target field to maintain the positiveness of the conductivity during the inverse process and to avoid penalizing small conductivity values. As shown in eq. (11), the expansion is composed from the sum of an infinite series. However, in practice, the expansion must be truncated into a finite number of terms, hereafter denoted as $p$,

$s=\sum_{i, j, k=0}^{p} a_{i j k} \Psi_{i}(x) \Psi_{j}(y) \Psi_{k}(z)$.

The high-order parts of polynomial expansion may increase quickly for large values of $x, y$ and $z$. As a result, $\Psi$ may excess to an extreme value that leads to truncation in the computation, especially in our case where the expansion is for a logarithmic-defined quantity (the electrical conductivity distribution). For this reason, the variables $x, y$ and $z$ should be normalized to length scales or/and multiplied with weight functions:

$s=\sum_{i, j, k=0}^{p} a_{i j k} \bar{\Psi}_{i}(\bar{x}) \bar{\Psi}_{j}(\bar{y}) \bar{\Psi}_{k}(\bar{z})$,

where $\bar{x}=x / L_{x}, \bar{\Psi}_{i}(\bar{x})=\Psi_{i}(\bar{x}) w(\bar{x})$, with $w$ being the weight function and $L_{x}$ is the length scale in the $x$ direction; similar denoting for the $y$ and $z$ directions. Hereafter, instead of solving inversion problem to obtain the logarithm of the electrical conductivity, we will develop an inversion approach to determine the value of the polynomial coefficient $a_{i j k}$.

The Hermite polynomial is commonly used to construct an orthogonal basis to expand random variables or solve stochastic partial differential equations (Abbasi \& Gholami 2017). In this study, we apply the Hermite polynomial expansion to approximate the conductivity field. The higher order of polynomial expansion may improve the precision of solutions, but it also leads to a potential risk of high oscillations and an expensive computation cost in terms of time and memory. For this reason, we limit the expansion at the order of three, which corresponds to solve 64 variables $a_{i j k}$.

\subsection{Inversion problem}

The inversion process involves minimizing the objective function defined as following:

$L=\left(\boldsymbol{B}-\boldsymbol{B}_{\mathrm{obs}}\right)^{T} \boldsymbol{R}^{-1}\left(\boldsymbol{B}-\boldsymbol{B}_{\mathrm{obs}}\right)+\left(\boldsymbol{a}-\boldsymbol{a}_{\text {prior }}\right)^{T} \Gamma^{-1}\left(\boldsymbol{a}-\boldsymbol{a}_{\text {prior }}\right)$,

where $\boldsymbol{B}(n \times 1)$ is the norm of predicted magnetic field and $\boldsymbol{B}_{\mathrm{obs}}$ $(n \times 1)$ denotes the norm of magnetic observation data with $n$ is the number of measurements. $\boldsymbol{R}(n \times n)=\xi^{2} \boldsymbol{I}$ is the covariance matrix of the measurement errors, $\boldsymbol{I}$ is an identical matrix and the scalar $\xi$ is set equal to 0.05 as the standard deviation of the measurement. $\boldsymbol{a}(l \times l)$ is the vector of coefficient in the polynomial expansion of the electrical conductivity in the logarithmic scale with $l$ is the number of variables. The quantity $\boldsymbol{a}_{\text {prior }}$ denotes the prior information on the estimated values. This information could be independently derived from other geophysical and geological surveys (Linde et al. 2006). The quantity $\Gamma(l \times l)$ is the regularization matrix defined here as an identical matrix and the inversion process is initiated with the first guess of a. Note that the first component $a_{0}$ is equal to the electrical conductivity of the background area and other components equal zero to get a solution characterized by a small norm. Theoretically, problems with a few numbers of parameters usually do not require regularization. However, as the sensitivity matrix $\mathbf{J}$ has a multidimensional null space in our cases, the solution thus must be regularized to stabilize the inverse problem as shown in eq. (16) (Tikhonov \& Arsenin 1977).

We recall that the MMR method only provides an idea on the contrast of the spatial distribution of electrical conductivity of the investigated zone. Thus, the initialization of the inverse process with a different value to the electrical conductivity of the background does not impact the reconstruction of the geometry of the anomaly or the gradient of the distribution.

Solving the inverted problem relies on an iterative process to estimate the recent step $\boldsymbol{a}_{k+1}$ :

$\boldsymbol{a}^{k+1}=\boldsymbol{a}^{k}+\alpha \Delta \boldsymbol{a}$

where

$\Delta a=\left[\boldsymbol{J}^{T} \boldsymbol{R}^{-1} \boldsymbol{J}+\boldsymbol{I}\right]^{-1} \boldsymbol{J}^{T}\left(\boldsymbol{B}_{\mathrm{obs}}-\boldsymbol{B}\right)$

with $\alpha$ is a scalar estimated from minimizing the function $L$ associated with a line search scheme and $\boldsymbol{J}(m \times n)$ is the Jacobian matrix

$J_{i j}=\frac{\partial B_{i}}{\partial a_{j}}$

with $B_{i}$ is the measurement $i$ in $B$ and $a_{j}$ is component $j$ in $a$. The computation of $J$ adopts a numerical difference scheme that requires to run the forward model with a perturbation of each component $a_{j}$ in $a$. The perturbation is set to 0.001 in the simulation.

\subsection{Applications}

This section is dedicated to validating the method through five case studies, starting with a simple case and moving on to more and more complex cases. The tests are designed to simulate and to cover various geophysical studies. The first application aims to detect a single underground anomaly from the magnetic measurement acquired on the ground surface. This targeted anomaly is defined as a sphere buried in a homogeneous background that constructs a discontinuous conductivity field. In the second study, we deal with a continuous and heterogeneous electric conductivity field generated with a geostatistical code, which is commonly encountered in geoscience applications. In the third example, we disturb an electrical conductivity field with an injection of salt under the measurement stations. Here the electrical conductivity is an evolving field and varies in both time and space, and this application case aims to study the effectiveness of MMR to follow the movement of a conductive body that can be a contaminant or saltwater intrusion. The fourth synthetic test is designed to validate the algorithm in the field conditions before applying in a real case study. We employ the same arrangement with the real measurements that mimics the field topography, electrical setting and magnetic stations. We finally apply the inverse scheme for a real investigation to detect preferential paths of seepage occurring in an earthen embankment of a headrace channel leading to a power station. To highlight the advantages and limits of the approach, we perform on the second application a comparison of the inversion with chaos polynomial expansion and the classical method of grid discretization, and a case study on the impact of choice of the truncation order of the polynomial expansion on the inversion results.

To evaluate the difference between the estimation and observed data, we define the coefficient of determination as following:

$R^{2}=1-\frac{\mathrm{SSR}}{\mathrm{SST}}$ 


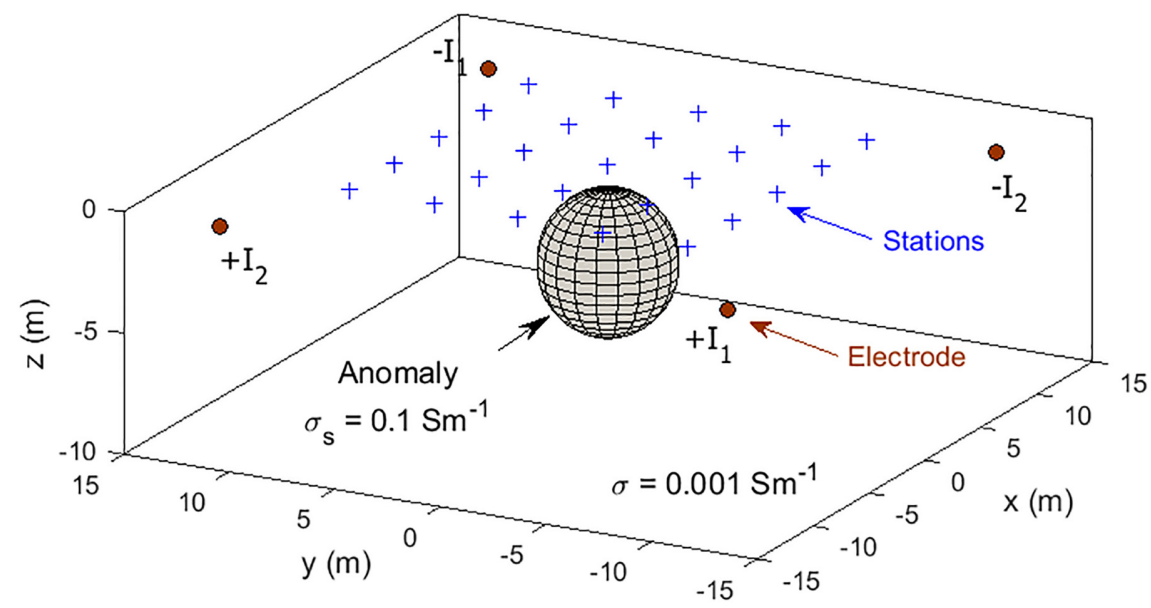

Figure 1. Geometry of the spherical anomaly with a conductivity of $\sigma_{\mathrm{s}}=0.1 \mathrm{~S} \mathrm{~m}^{-1}$ and background with a weak conductivity $\left(\sigma=0.001 \mathrm{~S} \mathrm{~m}^{-1}\right)$ for case study 1 . The brown dots denote the injection $\left(+I_{i}\right)$ and retrieval $\left(-I_{i}\right)$ electrodes of the electrical current, where the subscript $i$ indicates the measurement number. The blue crosses denote the position of the measurement stations where the three components of the magnetic fields are measured.

(a)

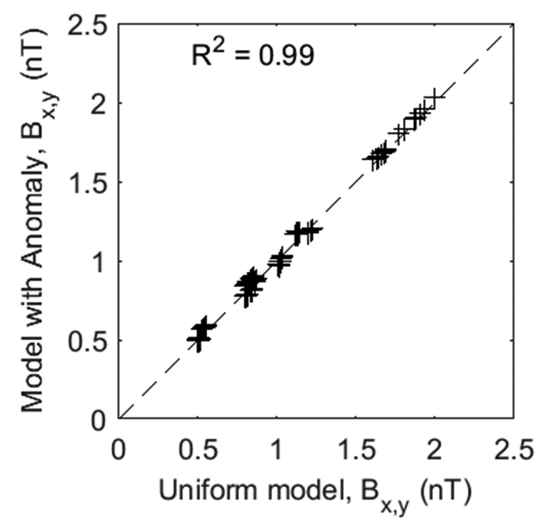

(b)

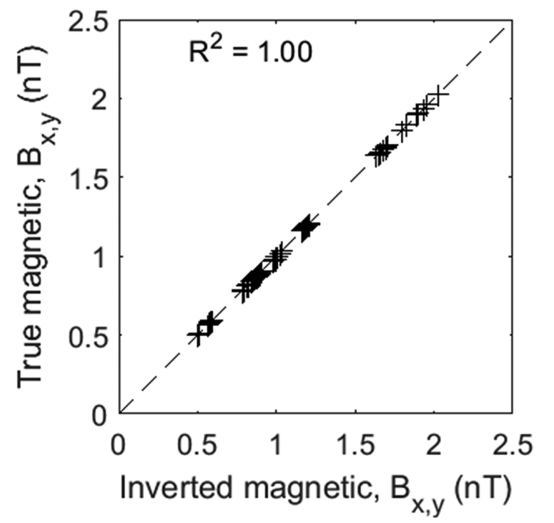

Figure 2. Comparison of the magnetic fields. (a) Comparison between the magnetic field components at the position of the measurement stations for the homogeneous ground (initial guess) and the magnetic field components with the presence of the conductivity anomaly in the ground. (b) Comparison between the true and inverted magnetic field data components.

where SSR $=\sum_{1}^{n}\left(B_{\mathrm{est}}-B_{\mathrm{obs}}\right)^{2}$ and SST $=\sum_{1}^{n}\left(B_{\mathrm{obs}}-\bar{B}_{\mathrm{obs}}\right)^{2}$ with $B_{\text {est }}$ and $B_{\text {obs }}$ denote the estimation and observed magnetic components, respectively, $\bar{B}_{\text {obs }}$ is the mean of $B_{\text {obs }}$ and $n$ denotes the number of observation data.

\subsection{Case study 1}

In the first case, we test the efficiency of the approach in uncovering of a single conductive anomaly buried in a homogeneous background. A spherical anomaly of radius $3 \mathrm{~m}$ is located within the medium at the depth of $4 \mathrm{~m}$ from the ground surface. The electrical conductivity of the sphere is $0.1 \mathrm{~S} \mathrm{~m}^{-1}$, which is more conductive than the surrounding environment characterized by a conductivity of $0.001 \mathrm{~S} \mathrm{~m}^{-1}$.

A primary electrical current is generated by the injection of $50 \mathrm{~mA}$ on two pairs of electrodes ( $\mathrm{A}$ and $\mathrm{B}$ ) installed at the depth $1 \mathrm{~m}$ from the ground surface; and 25 measurement stations are placed at the surface of domain to record the horizontal magnetic components (vertical component is neglected; see Fig. 1). The investigation has been conducted with two injections in which the electrodes of each pair are located at the two opposite corners and outside of the observation field. The analysis of the magnetic responses represents a margin difference between a model with the abnormal and homogeneous background (see Fig. 2a). This minor secondary anomaly is a challenge in the inversion process and its contamination by the noise may affect the quality of the prediction.

As mentioned above, the conductivity field is represented using the Hermite polynomial expansion of order three that corresponds to estimate 64 parameters in the inverse problem for 100 measurements of magnetic field. This makes the problem overdetermined. To initiate the inversion scheme, we use the value of the background electrical conductivity, starting therefore with a uniform model. The computation converges after four iterations and the predicted MMR in comparison with the true data is plotted in Fig. 2(b) where the estimation reproduces well the true magnetic field with a determination coefficient $R^{2}=1.00$. The inverted conductivity field associated with the true sphere is illustrated in Fig. 3. The heterogeneity in the medium is constructed in a spherical shape with some minor irregularity at the poles and along the equatorial region. However, the inverted conductivity field does not perfectly reproduce the true values of the conductivity anomaly. The issue may be due to the effect of the interpolation used in the parametrization when the polynomial series employed poorly describe the sharp change between the 


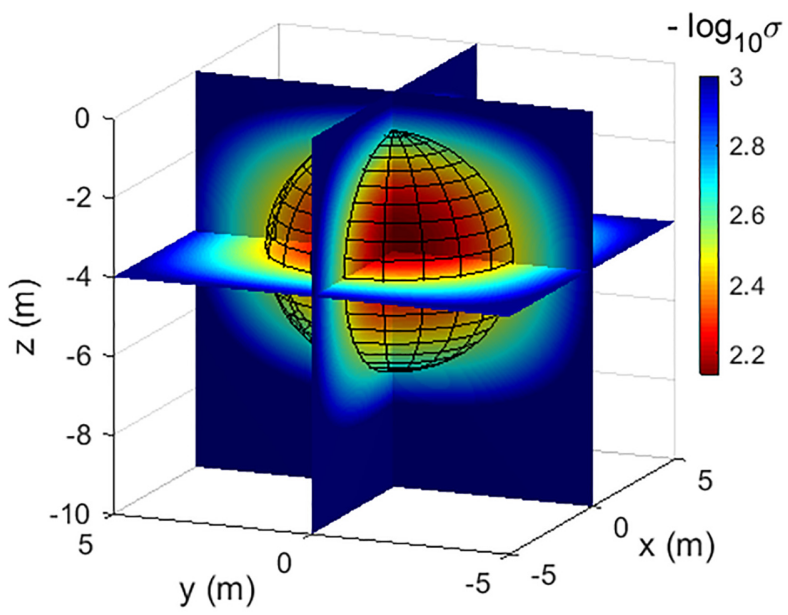

Figure 3. Comparison between the inverted conductivity field in logarithmic scale $\left(-\log _{10} \sigma\right)$ with the true spherical anomaly (black mesh, true model).

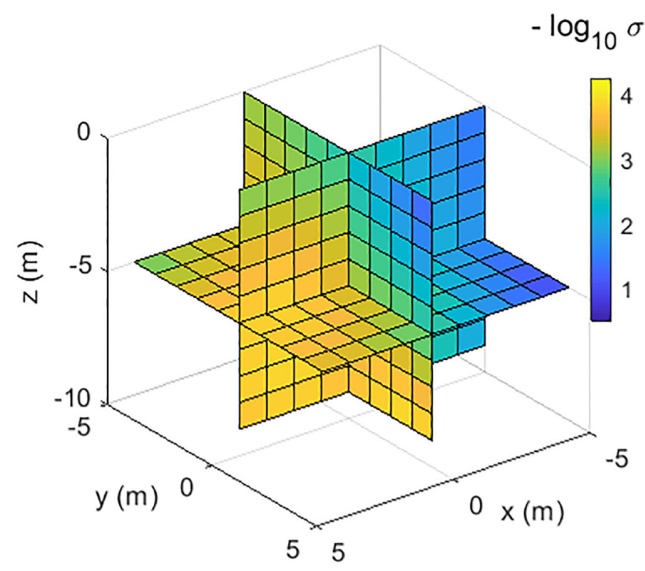

Figure 4. The true random conductivity field in logarithmic scale $\left(-\log _{10} \sigma\right)$ for case study 2 .

abnormal and its background. Nevertheless, the inverted solution can still provide useful information concerning the conductivity distribution of the subsurface. This point will be developed further in Sections 2.5 and 2.6.

\subsection{Case study 2}

We test now our algorithm for a more complex case in which a heterogeneous conductivity field is used to model the subsurface. A cube of $10 \times 10 \times 10 \mathrm{~m}$ locates right below the ground surface. The heterogeneity of this conductivity field is a four-order variation with a logarithmic range from -4.5 to -0.5 (see Fig. 4 ). The generation of the conductivity field follows a Gaussian distribution of which the sill and range are equal to 0.5 and $10 \mathrm{~m}$, respectively, employing the geostatistical approach in the SGMS software (Deutsch \& Journel 1997). This 3-D cube is buried in a uniform half-space of conductivity $0.005 \mathrm{~S} \mathrm{~m}^{-1}$ (equal to the mean of the random conductivity field) that is also used to reduce the impact of the boundary conditions.

We use the same observation array as in the previous case study (Fig. 5). The inverted process solves 64 coefficients of the polynomial expansion from calibrating 100 magnetic data observed on the surface. The algorithm converges after 22 iterations and the predicted conductivity field in logarithmic scale is plotted in Fig. 6(a). The comparison between the true input and the inversion conductivity in logarithmic scale is illustrated in Fig. 6(b) with an $R^{2}$ equal to 0.89 . The noise effect of the data measured is not considered in this study, however, such contamination may influence to the inverted solution quality.

The predicted field reproduces well the main aspect of the true heterogeneous conductivity in both terms of shape and values except some smoothness. The prediction seems to approach better the distribution of the recovered field in comparison to case study 1 when the Hermite polynomial expansion can interpret closely a continuous distribution. This improvement may contribute to the compatibility between the nature of the targeted conductivity field and the expansion series adopted. In this case, we apply the Hermit series to compose continuous polynomial functions, which properly approach better for a smooth distribution.

In the previous solution, we adopt the third order in the polynomial expansion. To further discuss the influence of choice of the truncation order on the inversion results, we solve the same problem using only two-order polynomials. This parametrization only makes 27 coefficients to estimate with respect to 100 measurements. The inverted conductivity field distribution is shown in Fig. 7(a). A comparison with the true conductivity field is plotted in Fig. 7(b). The prediction adequately follows the heterogeneities in the target medium, with lower precision compared to the inversion of the three-order polynomials as shown in Fig. 6.

Two-order polynomials provide a very smooth distribution of the electrical conductivity field. In the next test, we adopt four-order polynomials to reconstruct the conductivity field. The parametrization involves a total of 125 coefficients to be determined versus a total of 100 measurements or observations. Therefore, we work here with a slightly underdetermined problem instead of an overdetermined problem as done in the previous tests. That said, we can still apply the same solving scheme with an identical matrix in the regularization term. The inverted conductivity field is shown in Fig. 7(c) with a comparison to the true field in Fig. 7(d). Overall, the MMR prediction properly reproduces the subsurface conductivity. The algorithm can hence be adopted for both over- and underdeterministic problems.

To further validate our analysis, we perform an inversion using the classical grid-based representation of the unknown parameters for the same problem. The subsurface medium is discretized by grid of $10 \times 10 \times 10$ which results to 1000 variables to predict with 100 measurements as previous tests. Since the problem is strongly underdetermined, it requires the use of a constraint in regularization term. In this case, we opt for a geostatistical constraint by using the covariance applied in the generation of the true field. The inverted conductivity medium is illustrated in Fig. 8(a). The prediction reasonably follows variations of the conductivity field in the subsurface. However, the MMR grid-based solution also exhibits a significant loss of resolution with depth, which is detailed in Fig. 8(b). The inverted solution reproduces properly the upper half of the medium while the lower half is poorly reconstructed. Such inaccuracy in this case seems to be higher than our recent algorithm solution. This difference may reflect that the polynomial approach globally works when it approximates the whole medium with the same discrete function, which differs from a grid-based approach. The accuracy of upper part may then contribute to constrain the reconstruction of lower part. In the next case studies, we discuss further the resolution of MMR with depth. 


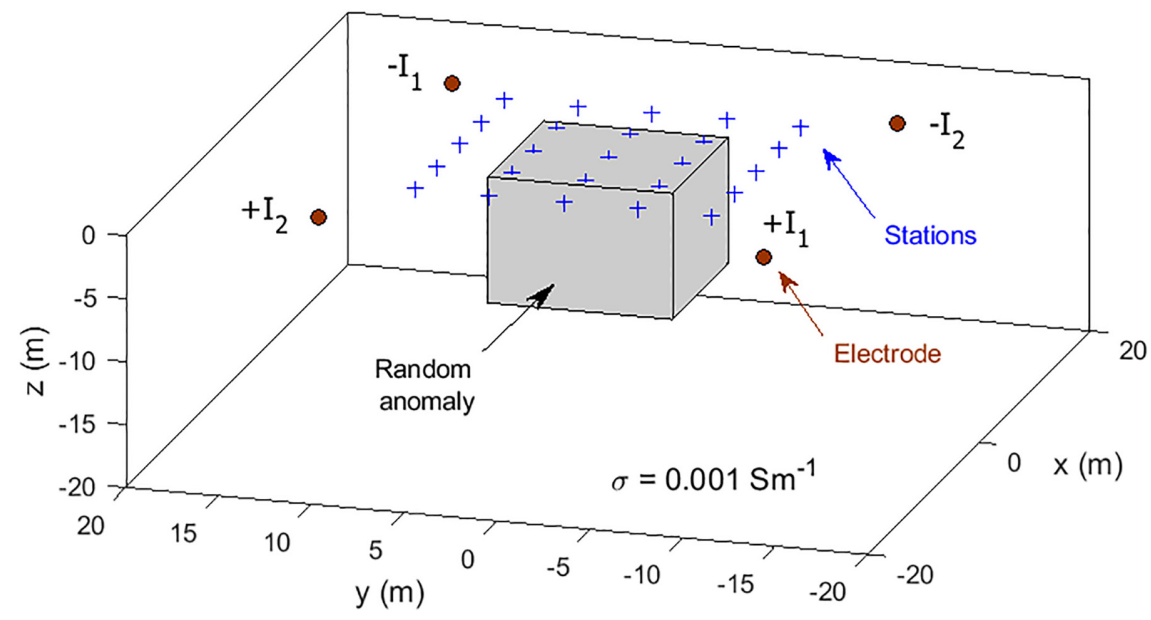

Figure 5. Position of the domain (grey area) for case study 2 where the heterogeneous electrical conductivity distribution shown in Fig. 4 is assigned. This domain is buried in a background conductivity with a conductivity of $\sigma=0.001 \mathrm{~S} \mathrm{~m}^{-1}$. The brown dots denote the injection $\left(+I_{i}\right)$ and retrieval $\left(-I_{i}\right)$ electrodes of the electrical current, where the subscript $i$ indicates the measurement number. The blue crosses denote the position of the measurement stations where the three components of the magnetic fields are measured.

(a)

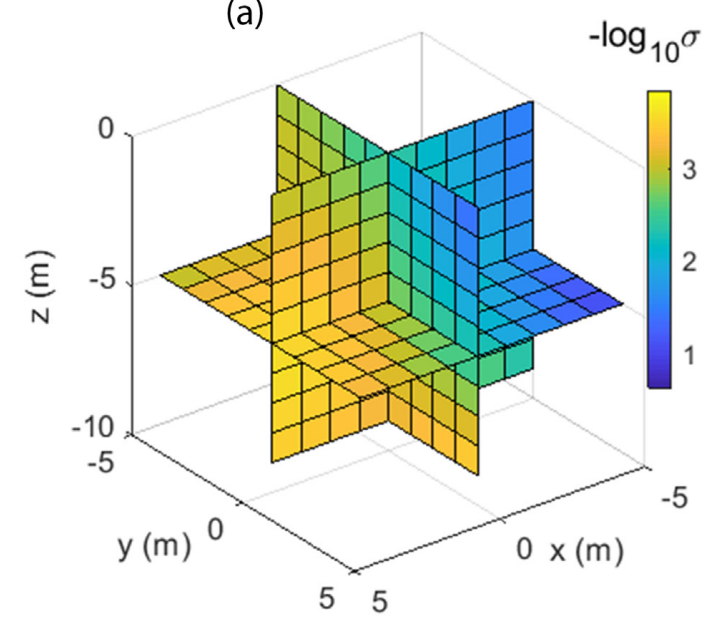

(b)

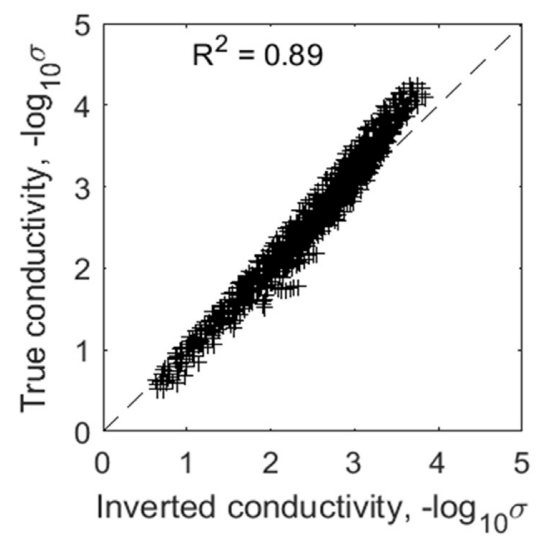

Figure 6. The inverted conductivity in logarithmic scale $\left(-\log _{10} \sigma\right)$. (a) Inverted field. (b) Comparison between the true and inverted conductivity fields.

\subsection{Case study 3: monitoring a salt plume migration}

In this study, we attempt to track the evolution of a conductivity field due to an injection of the saline solute through a well installed underground (see Fig. 9). The perturbation in the electrical conductivity associated with the migration of the salt tracer will be monitored adopting the inversion process.

The initial electrical conductivity of a saturated soil is given by (e.g. Waxman and Smits 1968)

$\sigma=\frac{\sigma_{f}}{F}+\sigma_{s}$,

where $F=\phi^{-\mathrm{m}}(-)$ is the electrical formation factor, which depends on the porosity of the medium ( $\phi$, unitless), $\sigma_{f}$ denotes the pore water electrical conductivity (in $\mathrm{S} \mathrm{m}^{-1}$ ) and the powerlaw (cementation or porosity) exponent $m$ (unitless) ranges from 1.3 to 2.5 (Revil et al. 1998). In this test, we suppose $\phi=0.3$, $k=1.5$ to yield $F=6.086$. The surface conductivity $\sigma_{s}$ (in $\mathrm{S}$ $\mathrm{m}^{-1}$ ) corresponds to the conductivity of grains, which is supposed to be constant in the whole aquifer and has the value of $0.005 \mathrm{~S} \mathrm{~m}^{-1}$.
The pore water conductivity is also dependent on the solute concentration (Jardani et al. 2013):

$\sigma_{f}=a c\left(\beta^{+}+\beta^{-}\right) e$,

where $c$ is saline concentration of the pore water (in $\mathrm{mol} \mathrm{m}^{-3}$ ), $\beta^{+}$ $\left(25^{\circ} \mathrm{C}\right)=5 \times 10^{-8} \mathrm{~m}^{2} \mathrm{~s}^{-1} \mathrm{~V}^{-1}$ and $\beta^{-}\left(25^{\circ} \mathrm{C}\right)=7 \times 10^{-8}$ $\mathrm{m}^{2} \mathrm{~s}^{-1} \mathrm{~V}^{-1}$ are the mobility of the cations and anions in the pore water, respectively. $e$ is the elementary charge of the electron $\left(e=1.6 \times 10^{-19} \mathrm{C}\right)$ and $a$ denotes a coefficient to convert the unit of concentration from $\mathrm{kg} \mathrm{m}^{-3}$ to $\mathrm{mol} \mathrm{m}^{-3}$.

We simulate an injection of saline water into a homogeneous ground from a well. The injection rate is constant of $1 \mathrm{~m}^{3} \mathrm{~d}^{-1}$ $\mathrm{m}^{-1}$. It locates at the depth from -2 to $-5 \mathrm{~m}$. We employ saline as the contaminated source with a constant concentration of $1 \mathrm{~mol}$ $\mathrm{m}^{-3}$. The test occurs continuously in $20 \mathrm{~d}$ and the magnetic field is observed instantaneously at 5,10 and $20 \mathrm{~d}$ from the beginning of saline injection. The inversion process is designed to track the evolution of the conductivity field.

We actually focus on the change in the electrical conductivity, and we consider the transport properties of the tracer to be known 


\section{Inverted}

(a)

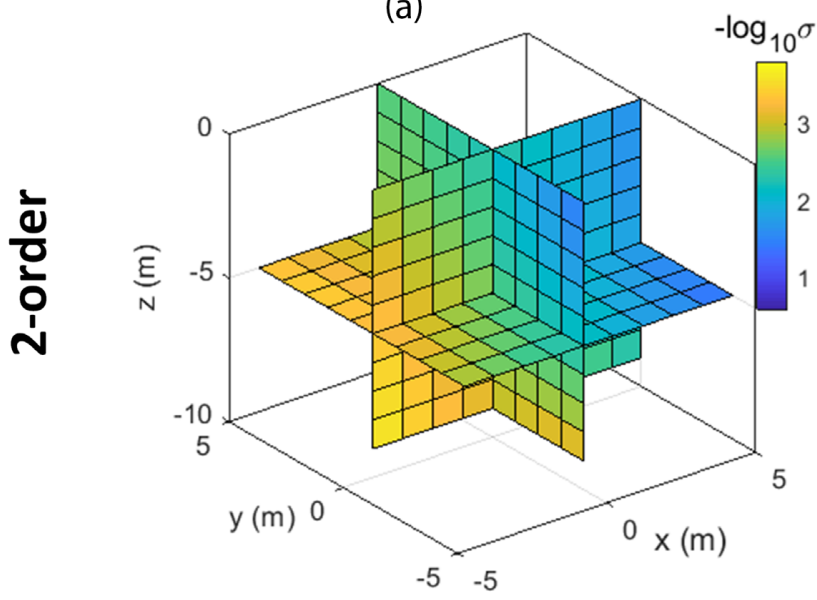

(c)

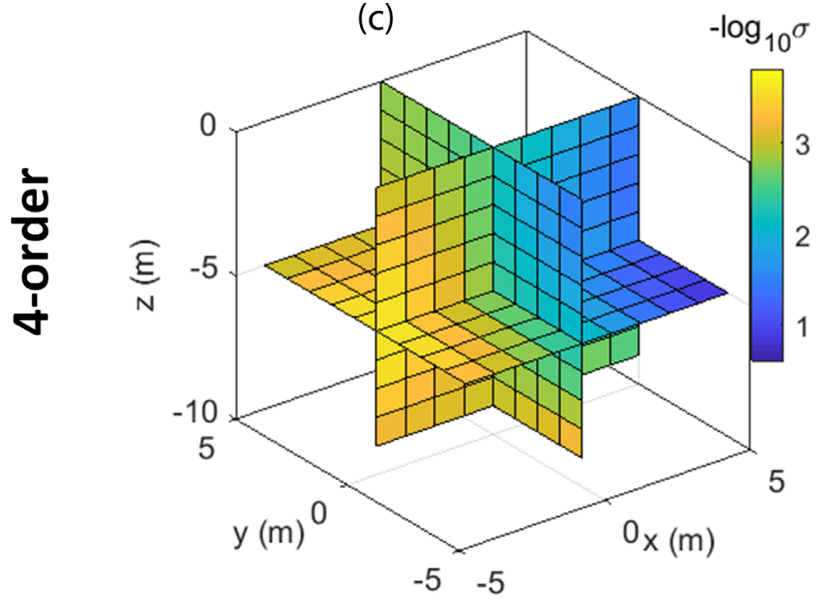

\section{Comparison}

(b)

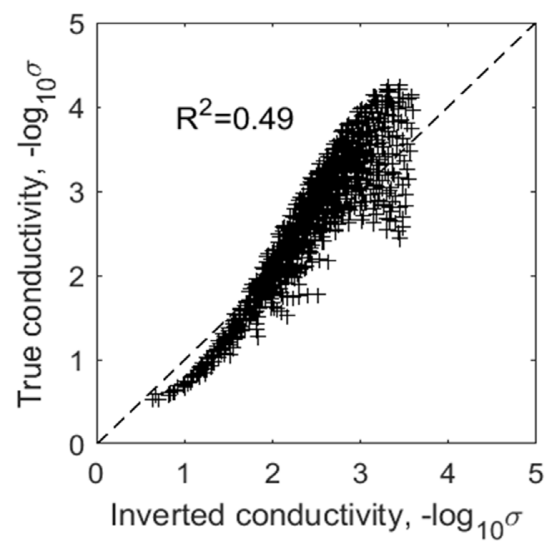

(d)

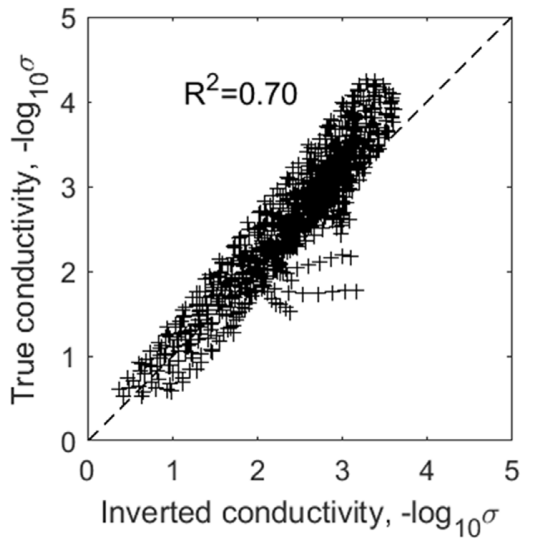

Figure 7. Inversion results for various polynomial expansion orders. By row, from top to bottom for two-order (a and b) and four-order (c and d) polynomials. By column, from left to right: inverted field and comparison to the true conductivity field.

Inverted

(a)

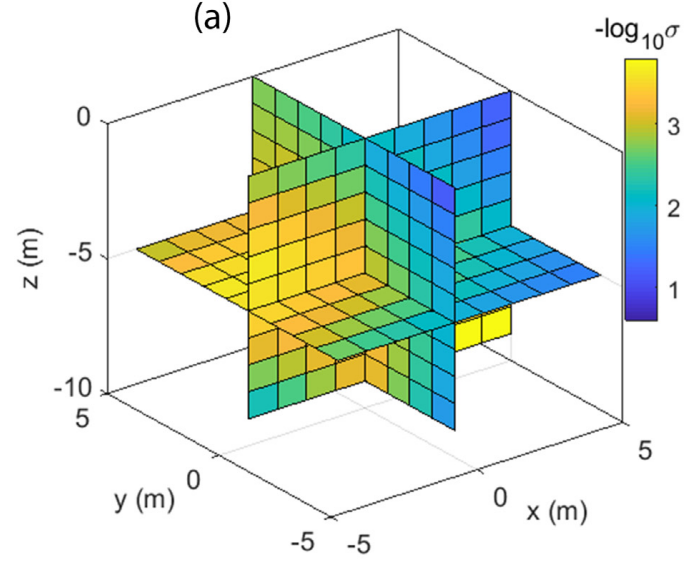

Comparison

(b)

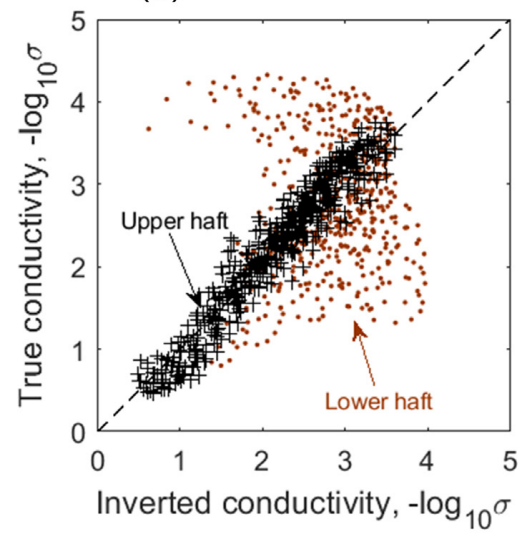

Figure 8. The inverted conductivity in logarithm scale $\left(-\log _{10} \sigma\right)$ using a grid discretization-based solution. (a) Inverted prediction. (b) Comparison to the true conductivity. Inverted result shows the evolution of the resolution of MMR with depth. We clearly see that the prediction accuracy of the upper haft (black crosses) is better comparing to one of the lower haft (red points). 


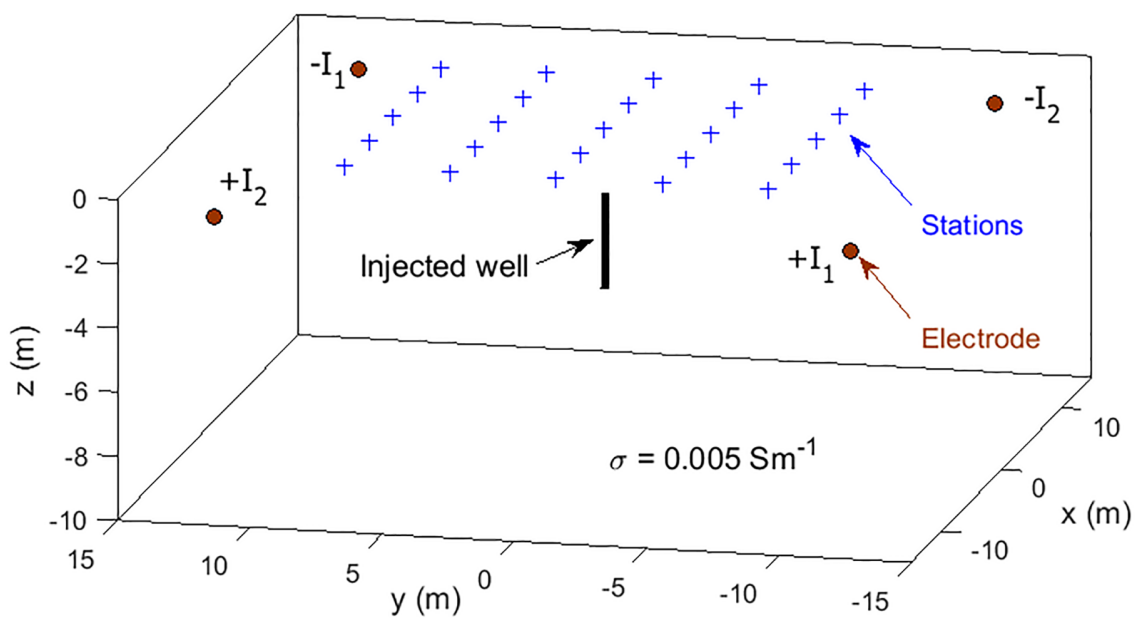

Figure 9. Configuration of the saline injection including the position of the injection electrodes and measurements for case study 3 . The brown dots denote the injection $\left(+I_{i}\right)$ and retrieval $\left(-I_{i}\right)$ electrodes of the electrical current, where the subscript $i$ indicates the measurement number. The blue crosses denote the position of the measurement stations where the three components of the magnetic fields are measured.

and controlled by the advection phenomena. The other processes, such as reaction, adsorption or absorption, are excluded from the transport equation. The flow obeys the Darcy equation with a homogeneous hydraulic conductivity of $10^{-4} \mathrm{~m} \mathrm{~s}^{-1}$ and porosity of 0.30 . The longitudinal dispersity is $\alpha_{L}=10 \mathrm{~m}$ and the transverse dispersity is $\alpha_{T}=3 \mathrm{~m}$. We solve the system to obtain the concentration field in space and in time. The corresponding conductivity field is converted using eq. (13), which is the target anomaly in the inversion process.

The inversion scheme applies the same observation arrangement as in the previous cases, which consists of an array of 25 stations and two pairs of current electrodes. The measurement stations are located on the surface around the injection well. We assume that the magnetic components are observed instantaneously at the time stage desired, which include 100 data for each set of data. In other words, the time required to get the data during a survey is much shorter than the time required to change the conductivity distribution in the subsurface by advection of the salty water. At each corresponding stage, we employ a three-order expansion of the conductivity field in three orthogonal directions. There are 64 coefficients to determine. Since the number of observations is larger than the number of model parameters, we are dealing with an overdetermined problem.

The injection of saline into the ground leads to an accumulation of salty water in the vicinity of the well. This accumulation gradually expands around due to the transport of the solute by advection and dispersion in the hydraulic conductivity field. We focus on the region of $5 \mathrm{~m}$ around the well covering the area with string changes in the electrical conductivity field. The result at $5 \mathrm{~d}$ after the injection is illustrated in Figs 10(a)-(c). This figure shows the true perturbation of the conductivity field, the inverted solution, and the comparison between them.

At the beginning of the injection, the perturbation almost locates in the close vicinity of the well with a resistivity of around $25 \Omega \mathrm{m}$. This value represents a sharp reduction from an initial (background) value of $200 \Omega \mathrm{m}$. The inverted estimation reproduces well the tendency of the field variation, the inverted result shows a minor smoothness at the injected well where the change is sharpest. Since the perturbation strongly localizes in a narrow area, such small anomaly will raise a difficulty in accessing by our expansion series which favour a smooth target field therefore without discontinuities.
The result at the $10 \mathrm{~d}$ injection is shown in Figs 10(d)-(f) where the contaminated area expanses associating with more perturbation in the conductivity field. The inverted field reconstructs well both shape and values of the electrical conductivity in the targeted area. Due to the transport of salt, the conductivity gradually decreases far away, while the accumulation of solute in the centre area shows only a slight increment in the conductivity field. Once the disturbance is less-localized around the well, the inverted estimation seems to improve in providing a higher correlation coefficient to the true measurement with $R^{2}=0.97$ comparing to $R^{2}=0.58$ of the previous stage at $5 \mathrm{~d}$ after the injection. A similar aspect can be observed in the data at $20 \mathrm{~d}$ of injection (Fig. 10). In the vicinity of the source, the highest conductivity reaches $0.08 \mathrm{~S} \mathrm{~m}^{-1}$ when the contaminated solute attains the source concentration. The inverted prediction shows a good agreement with the true observations $\left(R^{2}=0.95\right)$. Overall, the inversion results reconstruct well the evolution in the subsurface except some loss of resolution at the bottom of the medium. This example proves that the recent approach can be adopted to track the evolution of a conductive tracer within a medium in space over time.

\subsection{Case study 4: accounting for the effect of topography}

To imitate the behaviour of the process in a real condition, we reproduce the field condition on a synthetic case by using: real topography field, spatial positions of the measurements, shape of electrical wire route and the location of injection electrodes. The example serves as a validation aiming to test the effectiveness of the algorithm before adopting it in the real observations.

The MMR data on the ground surface are collected in situ covering an area of about $200 \mathrm{~m} \times 50 \mathrm{~m}$ as shown in Fig. 11 and the outline of the site is illustrated in Fig. 12. The equipment is arranged over an area of around $800 \mathrm{~m} \times 800 \mathrm{~m}$ to investigate a field of about $200 \mathrm{~m} \times 50 \mathrm{~m}$. A DC current $\left(I_{0}=1.5 \mathrm{~A}\right)$ flows between bipoles A and $\mathrm{B}$ to generate the magnetic response. The current electrodes are set down to a depth of $-1.5 \mathrm{~m}$ in the subsurface. The presence of electric current in the wire lying along the ground surface generates also a magnetic field. To consider and simulate this spurious effect, the whole current wires is taken into account in the modelling of the magnetic field. 
True

(a)

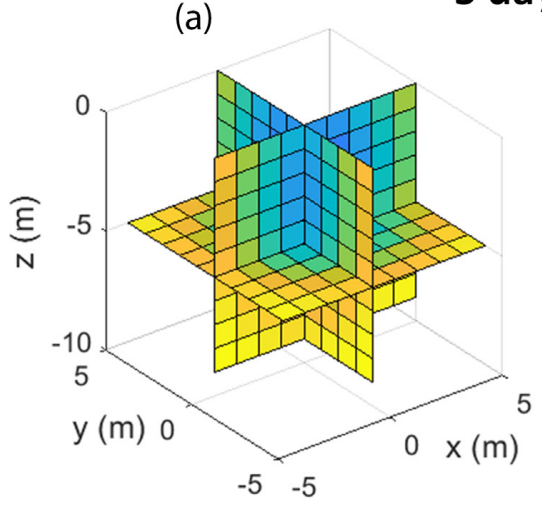

Inverted

(b)

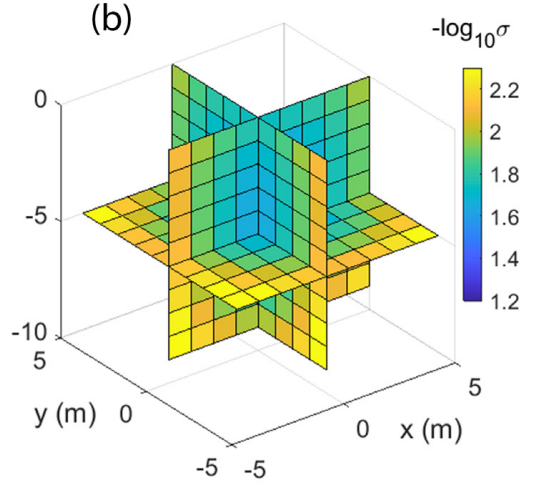

10 days
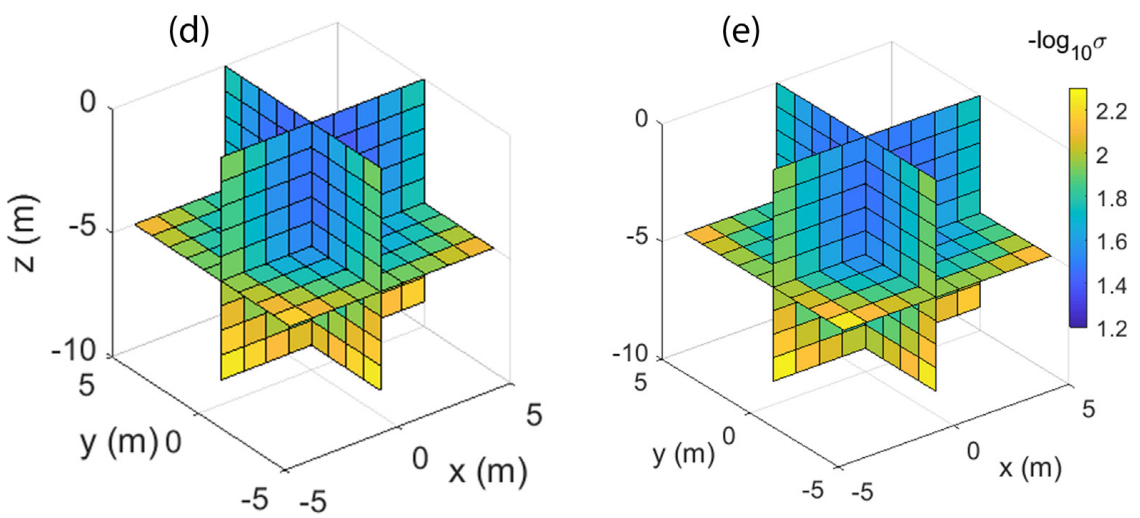

Comparison

(c)

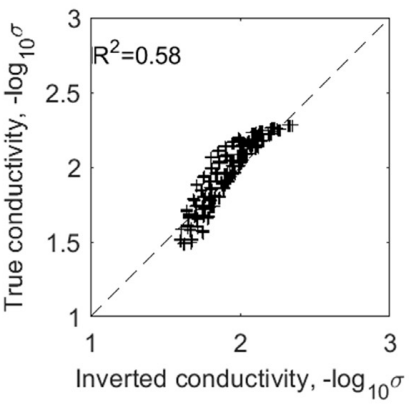

(f)

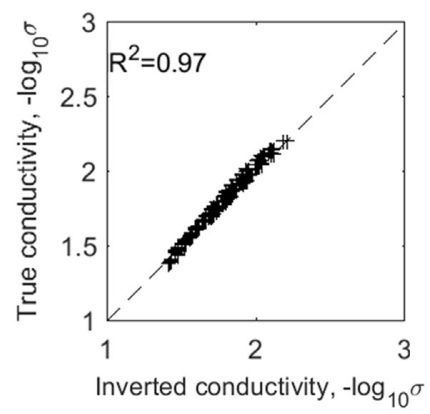

(i)

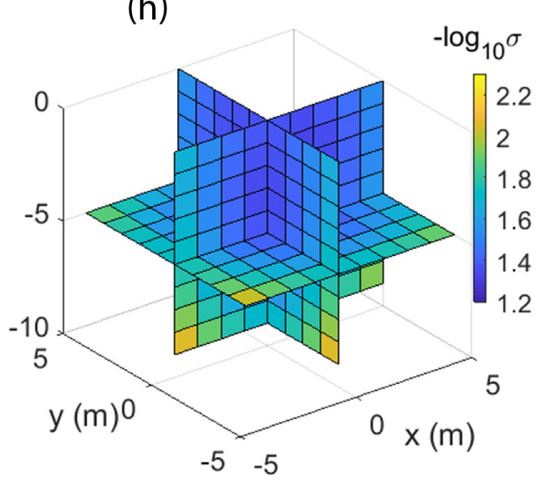

Figure 10. Conductivity evolves in time and space due to the saline injection. By column, from left to right: $(\mathrm{a}, \mathrm{d}, \mathrm{g})$ true conductivity, (b,e,h) inverted estimation and (c,f,i) comparison between them. By row, from top to bottom for data at time: (a-c) $t=5 \mathrm{~d}$, (d-f) $10 \mathrm{~d}$ and (g-i) $20 \mathrm{~d}$.

The resulting magnetic flux density is observed by a set of stations located at the ground surface. The system consists of 361 measurements arranged along a regular grid of $3.5 \mathrm{~m}$ in the $x$-direction and $2.5 \mathrm{~m}$ in the $y$-direction. Theoretically, the three components of the magnetic flux density are recorded; however, since the vertical component is negligible comparing to the horizontal ones, our data only focus on the values of the norm of the two horizontal components.
In this test, we target a synthetic conductivity of which the logarithm random field is generated from Gaussian distribution functions $\gamma_{x, y}=1.5 \exp \left(-r^{2} / 150^{2}\right)$ for the $x$ - and $y$-directions (horizontal) and $\gamma_{z}=1.5 \exp \left(-r^{2} / 50^{2}\right)$ for the $z$-direction (vertical) with $r$ is the distance. The conductivity field exhibits a conductive channel appearing in the shallow middle part of the investigated subsurface (see Fig. 13). This superficial anomaly is tagged as anomaly A1 below. It extends in an area of around $50 \mathrm{~m}$ long to a depth of $20 \mathrm{~m}$ 


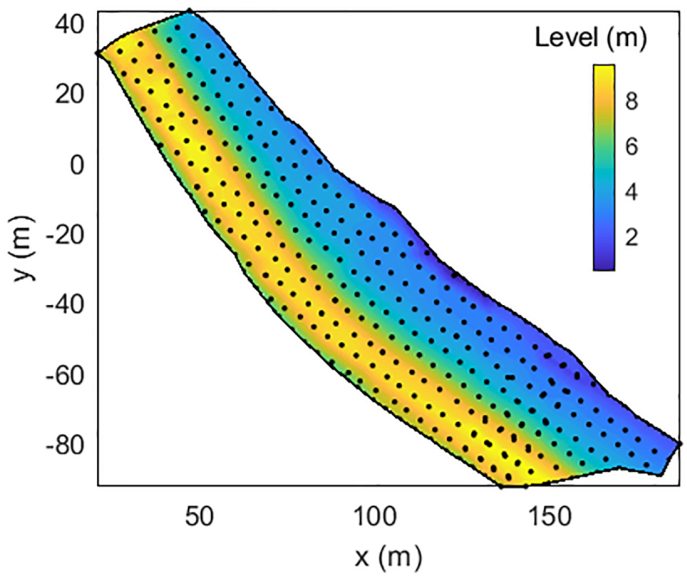

Figure 11. Topography of the field and the position of observation stations (black dots) for case study 4 . with an average conductivity of $0.05 \mathrm{~S} \mathrm{~m}^{-1}$. Two other anomalies characterized by less conductive areas locate at the depth of $30 \mathrm{~m}$ as marked in Fig. 13. One locates right below the anomaly A1 with an average conductivity of $10^{-4} \mathrm{~S} \mathrm{~m}^{-1}$, denoted as the anomaly A2. Another smaller anomaly is located at the limit of the measurement field (anomaly A3, see Fig. 13).

The numerical model is computed on a region $1800 \mathrm{~m} \times 1800 \mathrm{~m} \times 100 \mathrm{~m}$ with the real topography; however, the investigated area is around $250 \mathrm{~m} \times 100 \mathrm{~m} \times 50 \mathrm{~m}$ with a mean electrical conductivity of $10^{-3} \mathrm{~S} \mathrm{~m}^{-1}$. The targeted area is emerged in a half-space of a uniform conductivity $10^{-3} \mathrm{~S} \mathrm{~m}^{-1}$ as a boundary condition in the simulation. The forward problem is implemented in the COMSOL and the inversion process is solved in MATLAB. We approach the logarithm of the conductivity field by using the Hermite series with three-order polynomials. This corresponds again to 64 model parameters.

The inversion process starts with a uniform conductivity equal to the background value. High contrast in conductivity within the targeted zone is reflected in a significant anomaly in the magnetic observed on the ground surface in comparison to the relative response

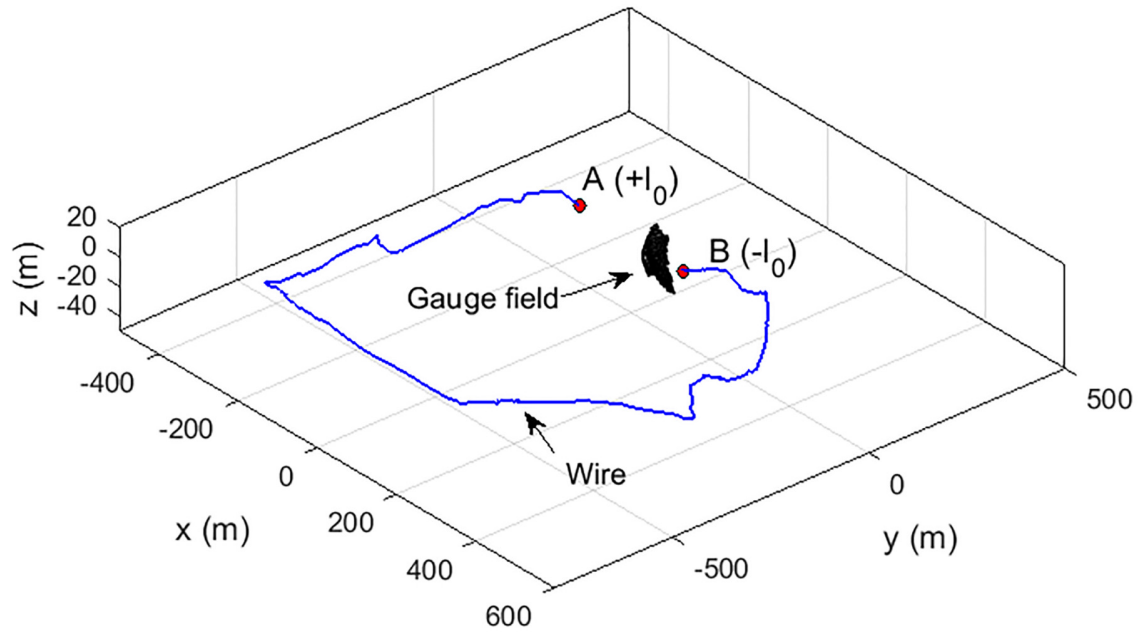

Figure 12. 3-D design to detect the anomaly, including the source/sink electrodes A and B, the wire (blue line) and the observation station (black dots).

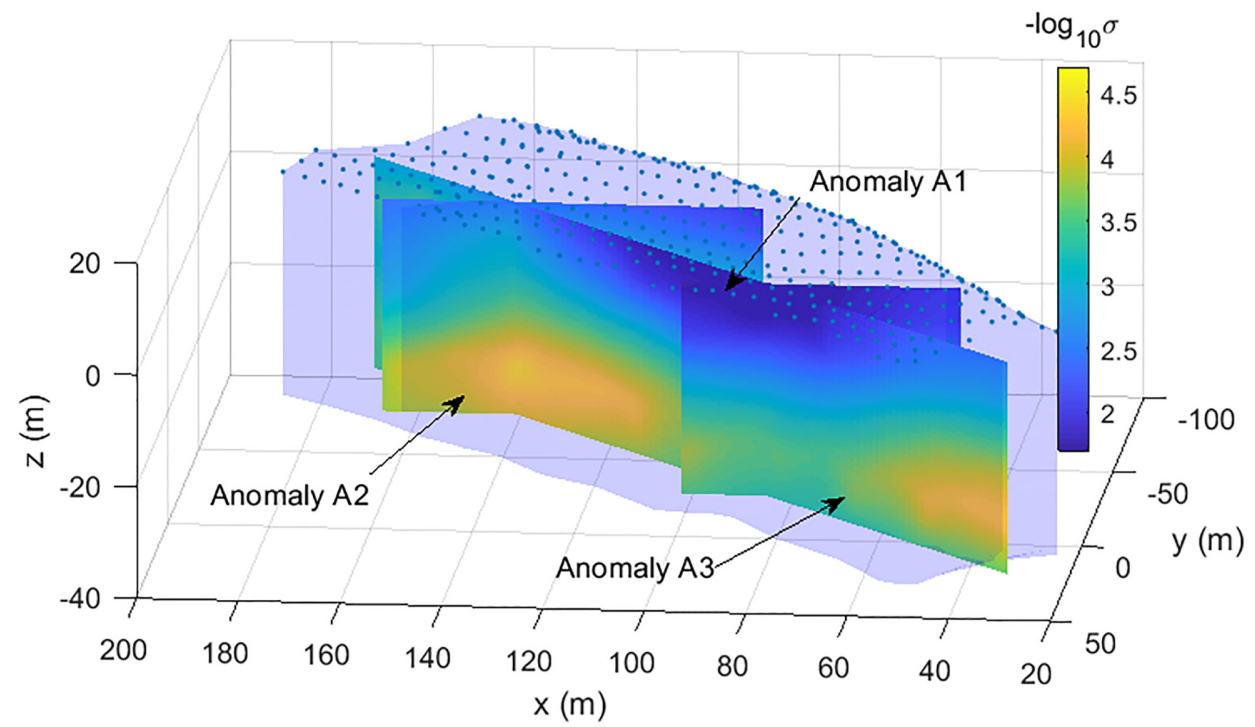

Figure 13. True conductivity field in logarithm, including three anomalies: more conductive anomaly (A1) and other two less conductive anomalies (A2 and A3). 
(a)

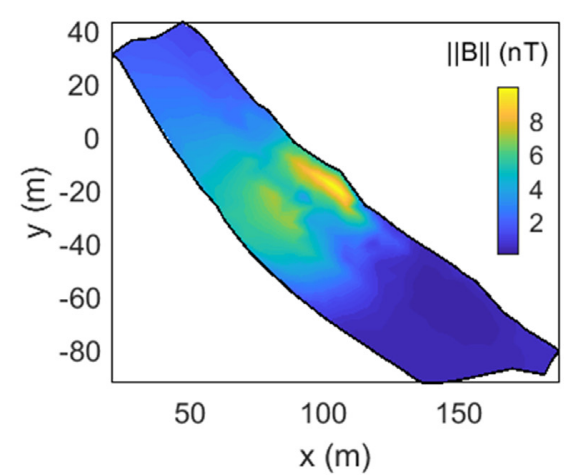

(b)

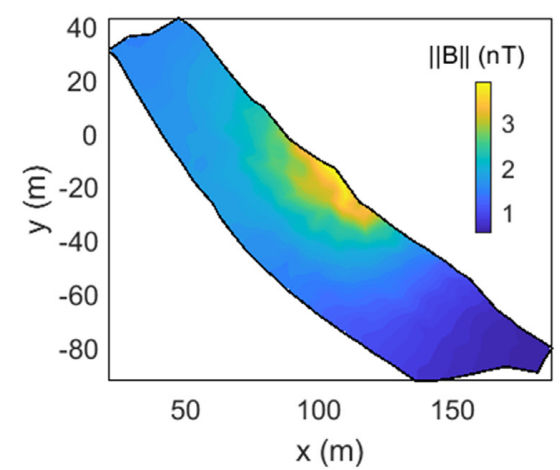

(c)

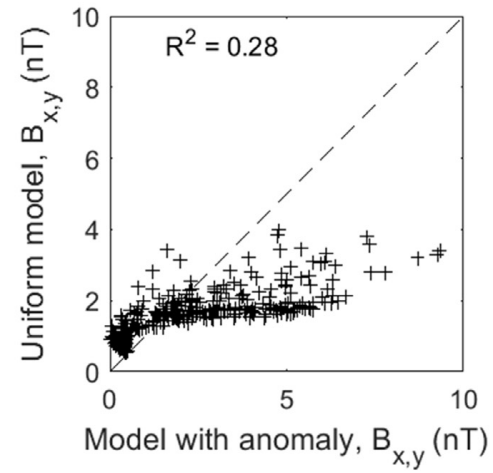

Figure 14. Magnetic field (in nT). (a) Observation data of the medium containing the anomaly. (b) Magnetic field corresponding to the initial homogeneous medium. (c) Comparison between them.

(a)

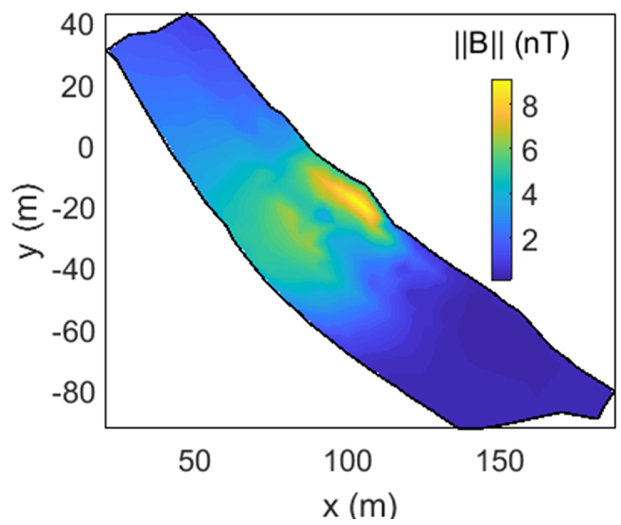

(b)

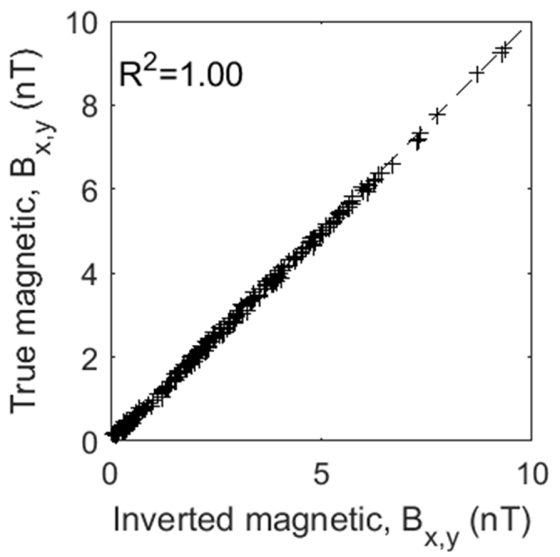

Figure 15. Magnetic field distribution (expressed in nT). (a) Inverted magnetic field distribution. (b) Comparison between the observations and the inverted magnetic field.

(a)

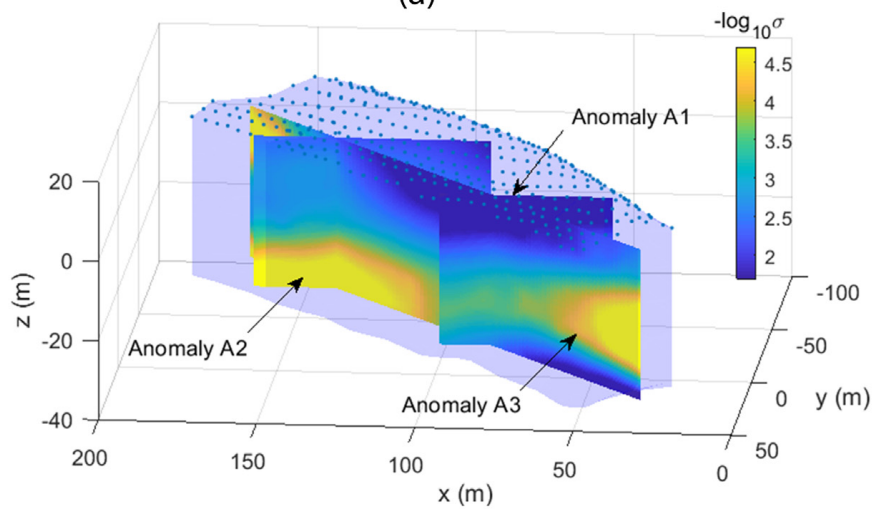

(b)

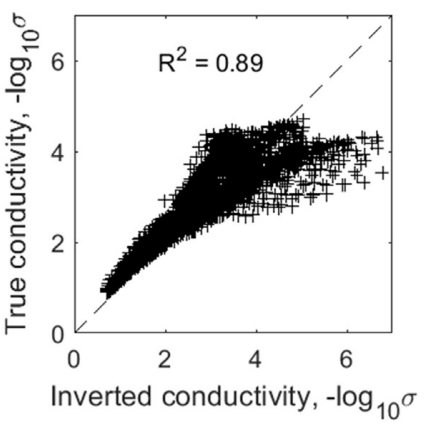

of the uniform model (see Fig. 14). Differences are shown in both shape and magnitude. The stronger variation in the shape of magnetic contour maps is clearly observed around the shallow anomaly A1. As demonstrated in previous studies, the deep anomalies often exhibit a narrow influence on the induced magnetic observed on the surface. The inversion process converges after 90 iterations and the inverted magnetic field is plotted in Fig. 15. The estimation resulted from the inversion reproduces well the observation magnetic field with $R^{2}=1.00$.

The inverted conductivity result is demonstrated in Fig. 16 with the comparison to the true field (down to $30 \mathrm{~m}$ depth from the ground surface). The estimation reproduces well the patterns in the medium including three anomalies, even though we have precision loss at the bottom boundary of around $40 \mathrm{~m}$ depth which corresponds to 


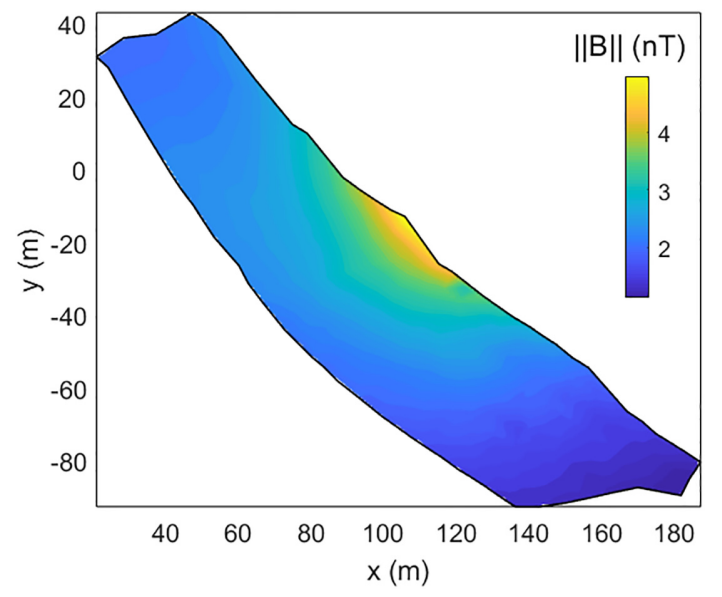

Figure 17. Observed magnetic field strength $\|B\|$ (expressed in nT).

the anomalies A2 and A3. Because the width of the gauged area is of only $50 \mathrm{~m}$ approximately, the bottom boundary is at the limit of the investigable range. A similar problem can be observed at other boundaries where some noises appear. Anomaly A3 also locates near at the edge of the survey area, its predicted location is slightly shallower than the true location.

Another point worth discussion is the degradation of resolution of solution with depth which is a typical limitation of many geophysical method such as: the electrical resistivity method, self-potential, gravimetry and magnetic methods. As shown in Fig. 16, the anomaly A1 near the ground surface is well reproduced in both terms of shape and value. However, the inverted tomography only constructs the shape of deeper anomalies, while the value of conductivity is poorly inverted; the error may excess orders of amplitude near the boundary. There are probably two reasons that contribute to this misinterpretation. First, it is usually more difficult to provide an accurate reconstruction of the deep area which locates far away from the monitoring stations installed on the ground surface because the primary field is still mainly focused in the shallow zone, and the deeper cells have a less impact on the inverse result. To tackle the problem of resolution in depth, some attempts have introduced using a depth-weighting function by giving increasing weight to voxels at increasing depth (i.e. Li \& Oldenburg 1996). However, this weighting function is inapplicable in our case where the parametrization is not defined by pixels or explicit location. Secondly, when the conductivity field is approached using a polynomial expansion, this approximation itself is a source of inaccuracy. Mathematically, this latter error depends on the correspondence between complexity of the targeted field and the expansion basis employed.

As discussed in Lu et al. (2015), a shortcoming of this projection approach is that the interpretation quality depends on adequacy of the statistic of the field to the choice of the polynomial basis. This limitation can be well-observed through comparing the result quality of previous examples. In the first three cases where the distribution of conductivity in the subsurface is Gaussian and rather simple, these fields fit to a polynomial projection, and consequently are correctly inverted. But this may not be always the case. To mitigate this shortcoming, it is promising to consider a hybrid scheme where this global smooth representation compromises with a local interpretation to compensate the smoothness. The local approach could be a gridded representation or B-splines. However, the solving system is more complicated in considering further assessment for the coupling representation.

\subsection{Field case study}

Now, we apply the algorithm to investigate the subsurface beneath a headrace canal containment embankment, with a specific purpose to delineate paths of groundwater flow leading to a spring where seepage is known to occur. The canal's embankments were constructed from boulder clay and gravel materials, lain directly on original ground surface after stripping away the organic matter including roots. The canal embankments were unlined; but the canal bed and sides (up to original ground level) were given $0.60 \mathrm{~m}$ of puddle lining where the canal was on porous soils.

The setting of the measurement field is the same as the previous synthetic case, except the real observation data are implemented to explore the real field. The current electrodes are arranged outside and in the centre line of the survey zone at the depth $-1.5 \mathrm{~m}$. The current source (A) is located in the headrace canal near the opposite embankment, and the sink (B) is installed some $30 \mathrm{~m}$ down-gradient of the embankment toe, close to the spring where seepage had been monitored. The two electrodes connect through an electric wire of about $2500 \mathrm{~m}$ length, arranged far away from the magnetic measurement stations to limit the interference of the current flowing in the power lines (Fig. 12). An AC electric current, at $380 \mathrm{~Hz}$, is generated at electrodes A and B with an amplitude of 1.5 A. Fig. 17 displays the norm of the recorded magnetic horizontal vectors at the ground surface. The magnitude of the magnetic field ranges from 0.75 to $5.25 \mathrm{nT}$. Since the electrode B is close to the observation area, it strongly influences the measurement in the down-gradient area, shaping a radial distribution in the data amplitudes.

The inversion process starts from an initial guess as a homogeneous conductivity field of $10^{-3} \mathrm{~S} \mathrm{~m}^{-1}$ change the final results). The magnetic field computed from this homogeneous model and its comparison to the observation are both illustrated in Fig. 18. The two magnetic fields are of the same form and magnitude order, such similarity somewhat implies that the conductivity distribution is not strongly heterogeneous in this area. The inversion converges after 63 iterations and the estimated magnetic contour map associated with the optimal conductivity field is plotted in Fig. 19 together with the observed data. The estimation reproduces well the form and magnitude of the magnetic field measured with a coefficient of $R^{2}=0.99$. The 3 -D conductivity field resulting from the inversion is shown in Fig. 20. A strong correlation between the inverted and observed data here, is better in comparison to one of sase study 4 with a similar condition, may contribute to the structure of the investigated field. In this case, the seepage confined in the middle of the subsurface as a single anomaly, which is easier than the previous complex case with three anomalies of various ranges.

The variation of electrical conductivity in the ground that corresponds to the seepage zone is characterized by a conductivity anomaly $\left(\sigma=0.01 \mathrm{~S} \mathrm{~m}^{-1}\right)$ that differs from the background value as shown in Fig. 20. Inverted results provide evidence of a potential ground water leakage from the headrace where the seepage path occurs and causes the spring downstream of the embankment. Following this 3-D prediction, we can localize the seepage path which should be closed by an existing grouting operation.

\section{Conclusion}

The MMR inversion technique developed in this paper can be used to invert 3-D conductivity field in the subsurface of the Earth. The targeted conductivity distribution is modelled by using a chaos polynomial expansion based on Hermite series. An advantage of the polynomial method is that the number of variables can be limited 
(a)

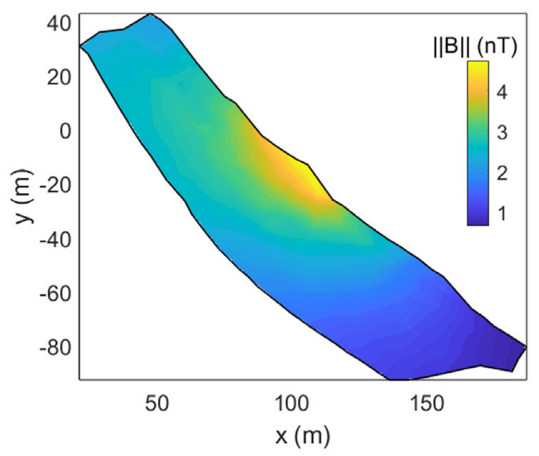

(b)

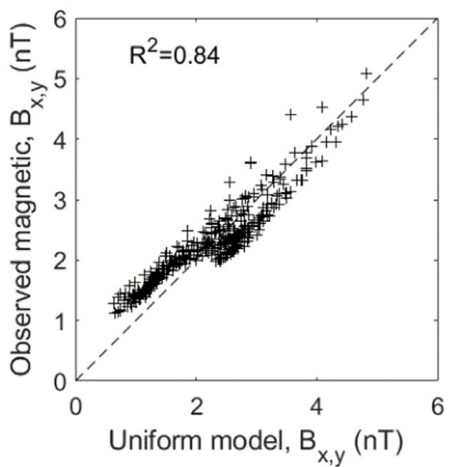

Figure 18. Magnetic field in a uniform half-space. (a) Magnetic field (in nT) associated with a uniform medium. (b) Comparison between the measured magnetic field and the magnetic field determined from a uniform half-space.

(a)

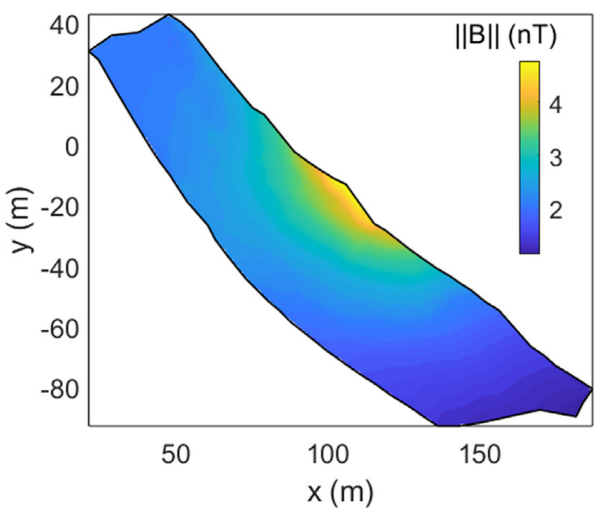

(b)

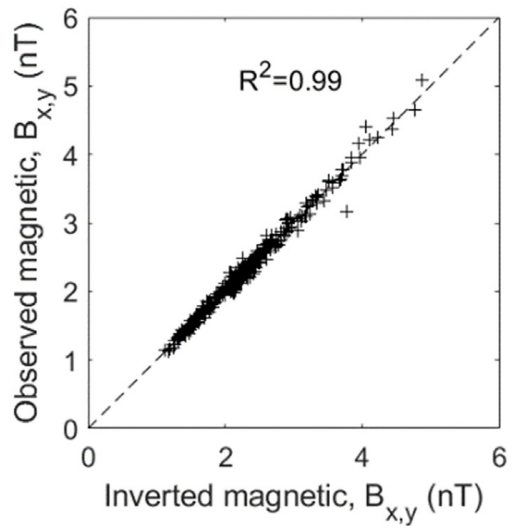

Figure 19. Inverted magnetic field. (a) Magnetic field. (b) Comparison with the observation data.

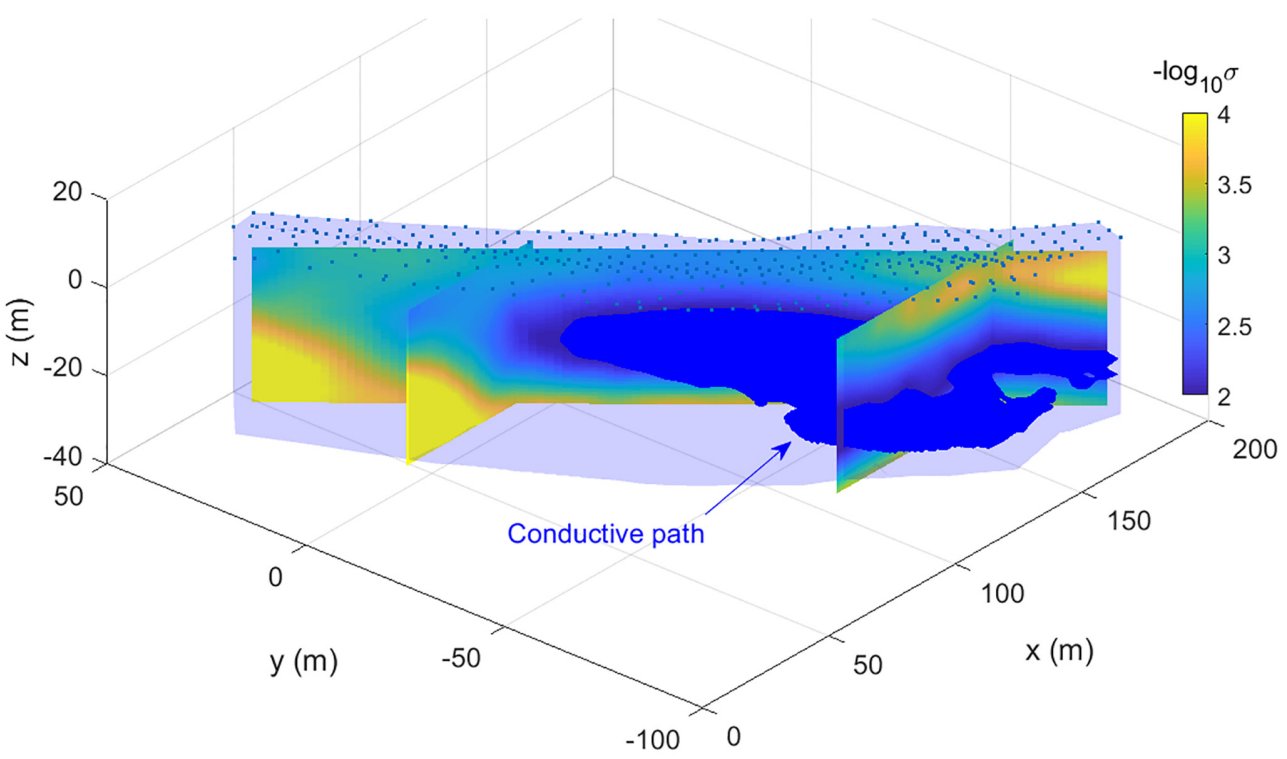

Figure 20. Prediction of logarithm of the conductivity field $\left(-\log _{10} \sigma\right)$, and the conductive path is presented by the blue surface (corresponding to a threshold of $\sigma=0.01 \mathrm{~S} \mathrm{~m}^{-1}$ ). 
by truncating the expansion of the variable. Such truncation reduces the variables inverted that helps to economize the input data required in the inverse process and create a chance to solve the problem in overdetermined condition. This approach is used to decrease the number of unknowns in the inverse problem and hence requires less observed data comparing to the conventional inversion process. The forward solving involves two steps. First, we determine the electric current density and then the corresponding magnetic field. Differential equations from these two steps are numerically solved with the finite-difference method. The inversion process is iteratively implemented, including a sensitivity matrix computation and the variables are estimated from a Gauss-Newton scheme. The proposed algorithm is tested with four synthetic cases. It is also applied to a real case study. Both discontinuous and continuous distributions of the conductivity field are investigated. The model can also consider the real setting of the field measurement including the electrical wire, sources and gauges, and topography.

As tested in this study, the inverted process is designed to tackle the heterogeneity in the targeted medium that aims to track both shape and conductivity value of the anomaly. The inverted results expose to well reproduce the heterogeneity, even though the quality of the prediction seems to depend on the structure of the targeted field. The polynomial expansion approaches better in a continuous medium of which both the distribution and range of conductivity value are properly predicted. For a discontinuous targeted field, where the anomaly exhibits a shape difference to the background, the recent polynomial approach can track the shape of the anomaly, however the value of heterogeneity is of a reasonable precision only. This limitation may relate to the nature of polynomial functions adopted in this study since the Hermite series approach better to smooth variations in the targeted field. The ill-posed nature of the inverse problem makes the interpretation of the data complex, which must be supplemented by prior information on the studied area. In these case studies, the solutions are strongly influenced by the choice of smoothness degrees that are controlled by the polynomial order and the initial model.

Ongoing work will extend the polynomial chaos approach to the more complex inverse problem. For instance, other polynomial series could be used to replace the Hermite expansion as in the recent work to better track the sharp change in the medium. To study a highly heterogeneous medium, an advanced scheme using a multiscale approach should be also considered in order to approach better the complexity of the medium and its statistics. Furthermore, by using the same expansion for conductivity field, the algorithm developed in this study is not only applicable for MMR but it can be also extended for inversion in ERT or for the joint inversion of ERT/MMR data.

\section{ACKNOWLEDGEMENTS}

We thank Willowstick Technologies Company for providing the field data. We thank the referee and the editor for fruitful discussions regarding our manuscript.

\section{REFERENCES}

Abbas, M., Jardani, A. \& Machour, N., 2018. Geophysical and geochemical characterisation of a site impacted by hydrocarbon contamination undergoing biodegradation, Near Surf. Geophys., 16(2), 176-192.

Abbasi, M. \& Gholami, A., 2017. Polynomial chaos expansion for nonlinear geophysical inverse problems, Geophysics, 82(4), R259-R268.
Binley, A. \& Kemna, A., 2005. DC resistivity and induced polarization methods, Hydrogeophysics, 50, 129-156.

Chen, J. \& Oldenburg, D.W., 2006. 3D inversion of magnetic induced polarization data. Explor. Geophys., 37(3), 245-253.

Chen, J., Haber, E. \& Oldenburg, D.W., 2002. Three-dimensional numerical modelling and inversion of magnetometric resistivity data. Geophys. $J$. Int., 149(3), 679-697.

de Boor, C., 1978. A Practical Guide to Splines, Springer-Verlag.

Debusschere, B.J., Najm, H.N., Matta, A., Knio, O.M., Ghanem, R.G. \& Le Mâtre, O.P., 2003. Protein labeling reactions in electrochemical microchannel flow: numerical simulation and uncertainty propagation, Phys. Fluids, 15(8), 2238-2250.

De Franco, R. et al. 2009. Monitoring the saltwater intrusion by time lapse electrical resistivity tomography: the Chioggia test site (Venice Lagoon, Italy), J. Appl. Geophys., 69(3), 117-130.

Deutsch, C.V. \& Journel, A.G., 1997. GSLIB: Geostatistical Software Library and User's Guide, 2 edn, Oxford University Press.

Edwards, R.N., 1974. The magnetometric resistivity method and its application to the mapping of a fault, Can. J. Earth Sci., 11(8), 1136-1156.

Edwards, R.N. \& Howell, E.C., 1976. A field test of the magnetometric resistivity (MMR) method, Geophysics, 41(6), 1170-1183.

Ezersky, M.G., Eppelbaum, L.V., Al-Zoubi, A., Keydar, S., Abueladas, A., Akkawi, E. \& Medvedev, B., 2013. Geophysical prediction and following development sinkholes in two Dead Sea areas, Israel and Jordan, Environ. Earth Sci., 70(4), 1463-1478.

Farra, V. \& Madariaga, R., 1988. Non-linear reflection tomography, Geophys. J., 95, 135-147.

Florsch, N., Llubes, M. \& Téreygeol, F., 2012. Induced polarization 3D tomography of an archaeological direct reduction slag heap, Near Surf. Geophys., 10, 567-574.

Fomel, S., 2000. Inverse B-spline interpolation, SEP Rep., 105, 79-108.

Friedman, S.P., 2005. Soil properties influencing apparent electrical conductivity: a review, Comput. Electron. Agric., 46(1-3), 45-70.

George, B.K., Torres-Verdín, C., Delshad, M., Siga, R., Zouioueche, F. \& Anderson, B., 2004. Assessment of in-situ hydrocarbon saturation in the presence of deep invasion and highly saline connate water, Petrophysics, 45, 141-156.

Ghanem, R., 1998. Probabilistic characterization of transport in heterogeneous media, Comput. Methods Appl. Mech. Eng., 158(3), 199-220.

Ghanem, R., Red-Horse, J.R. \& Sarkar, A., 2000. Modal properties of a space-frame with localized system uncertainties, in 8th ASCE Specialty Conference of Probabilistic Mechanics and Structural Reliability, Notre Dame, IN.

Grünhut, V., Bongiovanni, M. \& Osella, A., 2018, Using surface-downhole ERT for detecting contaminants in deep aquifers due to exploitation of oil reservoirs. Near Surf. Geophys., 16, 559-571.

Günther, T., 2007. DC2DInvRes - Direct Current 2D Inversion and Resolution, resistivity.net.

Jakosky, J.J., 1933. Method and apparatus for determining underground structure, US Patent No. US1906271A.

Jardani, A., Dupont, J.P. \& Revil, A., 2006. Self-potential signals associated with preferential groundwater flow pathways in sinkholes, J. geophys. Res., 111(B9).

Jardani, A., Revil, A. \& Dupont, J.P., 2013. Stochastic joint inversion of hydrogeophysical data for salt tracer test monitoring and hydraulic conductivity imaging, $A d v$. Water Resour., 52, 62-77.

Jessop, M., Jardani, A., Revil, A. \& Kofoed, V., 2018. Magnetometric resistivity: a new approach and its application to the detection of preferential flow paths in mine waste rock dumps, Geophys. J. Int., 215(1), 222-239.

Karaoulis, M., Kim, J.-H. \& Tsourlos, P., 2011. 4D active time constrained resistivity inversion, J. Appl. Geophys., 73(1), 25-34.

Kazakis, N., Pavlou, A., Vargemezis, G., Voudouris, K.S., Soulios, G., Pliakas, F. \& Tsokas, G., 2016. Seawater intrusion mapping using electrical resistivity tomography and hydrochemical data. An application in the coastal area of eastern Thermaikos Gulf, Greece, Sci. Total Environ., 543, 373-387.

Kitanidis, P.K., 1998. Stochastic approaches to inverse problems, Trans. Ecol. Environ., 17, 281-288. 
Kulessa, B., Jaekel, U., Kemna, A. \& Vereecken, H., 2002. Magnetometric Resistivity (MMR) imaging of subsurface solute flow: inversion framework and laboratory tests, J. Environ. Eng. Geophys., 7(3), 111-118.

LaBrecque, D., Sharpe, R., Casale, D., Heath, G. \& Svoboda, J., 2003. Combined electrical and magnetic resistivity tomography: synthetic model study and inverse modeling, J. Environ. Eng. Geophys., 8(4), 251-262.

Le Mâtre, O.P., Knio, O.M., Najm, H.N. \& Ghanem, R.G., 2001. A stochastic projection method for fluid flow: I. Basic formulation, J. Comput. Phys., 173(2), 481-511.

Le Mâtre, O.P., Reagan, M.T., Najm, H.N., Ghanem, R.G. \& Knio, O.M., 2002. A stochastic projection method for fluid flow: II. Random process, J. Comput. Phys., 181(1), 9-44.

Li, Y. \& Oldenburg, D.W., 1996. 3-D inversion of magnetic data, Geophysics, 61(2), 394-408.

Liao, Q., Deng, Y., Shi, X., Sun, Y., Duan, W. \& Wu, J., 2018. Delineation of contaminant plume for an inorganic contaminated site using electrical resistivity tomography: comparison with direct-push technique, Environ. Monit. Assess., 190(4), 187, doi:10.1007/s10661-018-6560-3.

Linde, N., Binley, A., Tryggvason, A., Pedersen, L.B. \& Revil, A., 2006. Improved hydrogeophysical characterization using joint inversion of crosshole electrical resistance and ground-penetrating radar traveltime data, Water Resour. Res., 42, W12404, doi:10.1029/2006WR005131.

Loke, M.H. \& Barker, R., 1996. Rapid least-squares inversion of apparent resistivity pseudosections using a quasi-Newton method, Geophys. Prospect., 44(1), 131-152.

Lu, F., Morzfeld, M., Tu, X. \& Chorin, A.J., 2015. Limitations of polynomial chaos expansions in the Bayesian solution of inverse problems, J. Comput. Phys., 282, 138-147.

Marzouk, Y.M., Najm, H.N. \& Rahn, L.A., 2007. Stochastic spectral methods for efficient Bayesian solution of inverse problems, J. Comput. Phys., 224(2), 560-586.
Nabighian, M.N., 1984. Toward a three-dimensional automatic interpretation of potential field data via generalized Hilbert transforms: fundamental relations, Geophysics, 49(6), 780-786.

Pereyra, V., 1996. Modeling, ray tracing, and block nonlinear travel-time inversion in 3-D. Pure appl. Geophys., 148, 345-386.

Reagan, M.T., Najm, H.N., Debusschere, B.J., Le Maître, O.P., Knio, O.M. \& Ghanem, R.G., 2004. Spectral stochastic uncertainty quantification in chemical systems, Combust. Theory Modelling, 8(3), 607-632.

Redhaounia, B., Ilondo, B.O., Gabtni, H., Sami, K. \& Bédir, M., 2016. Electrical resistivity tomography (ERT) applied to karst carbonate aquifers: case study from Amdoun, Northwestern Tunisia, Pure appl. Geophys., 173(4), 1289-1303.

Revil, A., Cathles, L.M., III, Losh, S. \& Nunn, J.A., 1998. Electrical conductivity in shaly sands with geophysical applications, J. geophys. Res., 103(B10), 23 925-23 936.

Samouëlian, A., Cousin, I., Tabbagh, A., Bruand, A. \& Richard, G., 2005. Electrical resistivity survey in soil science: a review, Soil Tillage Res., 83(2), 173-193.

Tikhonov, A. \& Arsenin, V., 1977. Solutions of Ill-Posed Problems, Winston.

Vinegar, H. \& Waxman, M., 1984. Induced polarization of shaly sands, Geophysics, 49, 1267-1287.

Waxman, M. \& Smits, L., 1968. Electrical conductivities in oil-bearing shaly sands, Soc. Pet. Eng. J., 8(2), 107-122.

Wiener, N., 1938. The homogeneous chaos, Am. J. Math., 60(4), 897-936.

Xiu, D. \& Karniadakis, G., 2002. The Wiener-Askey polynomial chaos for stochastic differential equations, SIAM J. Sci. Comput., 24(2), 619-644.

Xiu, D., Lucor, D., Su, C.-H. \& Karniadakis, G., 2001. Stochastic modeling of flow-structure interactions using generalized polynomial chaos, $J$. Fluids Eng., 124(1), 51-59.

Zhang, N. \& Wang, Y., 2010. Scattered interpolation with multilevel Bsplines for seismic reconstructions, in 72nd EAGE Conference and Exhibition incorporating SPE EUROPEC 2010, Vol. 7, pp. 4936-4940. 